\title{
CRPS: Aktuelle Erkenntnisse und Empfehlungen aus der Forschung
}

\author{
Claudia Storz, Eduard Kraft
}

\section{In Punkto Prädiktion, Prävention und Therapie tut sich einiges in der CRPS-Forschung. Neue Ergebnisse räumen mit alten Mythen auf, vielversprechende präventive und therapeutische Ansätze werden entwickelt. So könnten irgendwann, bei ausreichender Datenlage, die Gabe von Vitamin C standardmäßig zur Prävention und Exergames zur Therapie des CRPS gehören.}

Das komplexe regionale Schmerzsyndrom (CRPS) ist eine Erkrankung mit vielfältigen Erscheinungsformen, die durch eine unterschiedliche Symptomkombination aus somatosensorischen, motorischen, autonomen und psychologisch-kognitiven Dimensionen geprägt ist. Diese Vielfältigkeit an Symptomen und Untersuchungsbefunden kann bei demselben Patienten innerhalb des Krankheitsverlaufes sehr variabel sein. Dementsprechend ist es notwendig, CRPS aus Sicht des biopsychosozialen Modells zu betrachten und mit einem modernen, integrierten multimodalen Konzept zu behandeln.

\section{Neue Erkenntnisse in Ätiologie und Pathogenese}

Eine eindeutige Ursache für das Entstehen eines CRPS ist bis dato noch unbekannt. Charakteristisch ist jedoch das Zusammenspiel von peripheren und zentralen pathophysiologischen Prozessen als überschießende Reaktion auf eine Verletzung. Frühere Konzepte, wie zum Beispiel, dass die sympathische Dysfunktion das vorherrschende Problem darstellt oder dass ein CRPS in (stereotypen) Stadien auftritt, sind inzwischen überholt. Ebenso gibt es keinen Zusammenhang zwischen dem Auftreten eines CRPS und einer Vorgeschichte mit schmerzhaften psychischen Problemen, einer Somatisierung oder dem Simulieren der Betroffenen [1][2][3].

In den meisten Fällen entsteht ein CRPS nach einer Verletzung einer Extremität. Dabei gibt es keinen nachweislichen Zusammenhang zwischen der Entstehung der Erkrankung und der Schwere des Traumas. So entsteht ein CRPS häufig nach banalen Verletzungen ( $>$ Abb. 1), wohingegen massive Traumata kein CRPS nach sich ziehen müssen. Ein hoher Schmerzwert eine Woche nach dem Trauma kann ein Indikator für ein Erkrankungsrisiko darstellen [4].
Folgende Aussagen zur Prophylaxe oder Verlaufsmilderung eines CRPS werden aktuell wissenschaftlich diskutiert:

- Eine frühzeitige und angemessene Rehabilitationsbehandlung direkt nach einer distalen Radiusfraktur kann die Entwicklung eines CRPS verhindern [5].

- Die Verwendung von Vitamin C nach distaler Radiusfraktur hat einen positiven Effekt auf eine mögliche Entwicklung eines CRPS [6].

- Die Gabe von Steroiden kann bei sehr frühzeitiger CRPS-Diagnose zur Verhinderung eines verlängerten Krankheitsverlaufs beitragen [7].

Bevor jedoch eine der aufgeführten Präventionsmöglichkeiten definitiv empfohlen werden kann, benötigt es aussagekräftige Studien, die diese Annahmen bestätigen.

\section{Die Bedeutung des Chronifizierungsprozesses}

Nach dem Auftreten eines akuten CRPS tritt in den meisten Fällen innerhalb eines Jahres eine deutliche Verbesserung ein. Es wird jedoch geschätzt, dass zirka $40 \%$ aller Patienten mit CRPS eine längerfristige Beeinträchtigung trotz dieser Verbesserung und der Ausheilung der Erkrankung erfahren. Zusätzlich ist bei 15-20\% der Patienten der Verlauf chronisch [8]. Bislang gibt es nur sehr wenige prädiktive Marker, zum Beispiel psychische Komorbiditäten wie hohes Angstempfinden, die eine Beurteilung des Chronifizierungsrisikos oder die Bestimmung des natürlichen Verlaufs der Erkrankung erlauben. Invasive Maßnahmen sowie zu späte oder schmerzhafte Therapieverfahren begünstigen zusätzlich die Chronifizierung der Erkrankung [9].

Patienten mit einem chronischen CRPS-Verlauf leiden neben den Schmerzen vor allem an den motorischen Funktionseinschränkungen und den daraus resultierenden Schwie- 


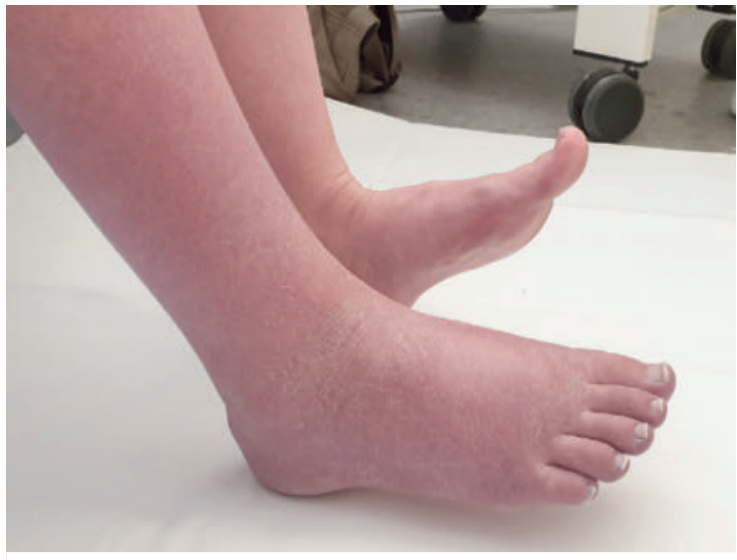

Abb. 1 CRPS des rechten Fußes nach einem Distorsionstrauma. (Quelle: C. Storz, E. Kraft)

rigkeiten im Alltags-, Berufs- und Privatleben [10][11]. Die Funktionseinschränkungen äußern sich meist in einem reduzierten aktiven Bewegungsumfang und einem erlernten Nichtgebrauch des betroffenen Körperteils ( $>$ Abb. 2). Auch die schmerzinduzierte Einschränkung und eine Kinesiophobie (Angst vor Bewegung) reduzieren zusätzlich den Einsatz der betroffenen Extremität im Alltag. Da CRPS zu den Erkrankungen zählt, die potenziell eine langfristige Beeinträchtigung nach sich ziehen, ist der Übergang vom akuten zum chronischen Stadium (ab 6-12 Monate) von großer klinischer und wissenschaftlicher Bedeutung.

Grundsätzlich gilt, dass für die Behandlung von Langzeitpatienten bis jetzt keine empfohlenen, evidenzbasierten Standards existieren. Meist profitieren sie trotzdem sowohl von der Unterstützung eines Selbstmanagements als auch von Behandlungsstrategien, wie sie auch bei allgemein chronischen Schmerzen zum Einsatz kommen [12][13].

\section{Veränderungen der kortikalen Zentren}

Beim CRPS wurden bereits multiple Veränderungen in kortikalen Zentren des Gehirns nachgewiesen. Es gibt Ergebnisse aus Studien, die relevante maladaptive neuroplastische Veränderungen der zentralen Repräsentation im sensomotorischen System zeigen [14][15]. Arbeiten von Di Pietro und Kollegen zeigen Veränderungen der kortikalen Darstellung der betroffenen Extremität in Form einer reversiblen Schrumpfung des somatosensorischen Kortex [16]. Eine Schrumpfung dieser Areale wäre eine überzeugende Erklärung für viele Wahrnehmungsstörungen. Aufgrund Mängel in den zugrundeliegenden Studien und einer wiederholten Auswertung neuerer Studien wurden diese Ergebnisse erweitert. So stellte sich bei der erneuten Auswertung heraus, dass Patienten mit CRPS mit einer vergrößerten Darstellung ihrer gesunden Extremität im sensomotorischen Kortex auffielen und nicht, wie erst angenommen, mit einer verkleinerten Darstellung der betroffenen Extremität [17]. Die genauen Veränderungen sind Gegenstand ständiger wissenschaftlicher Kontroversen und es

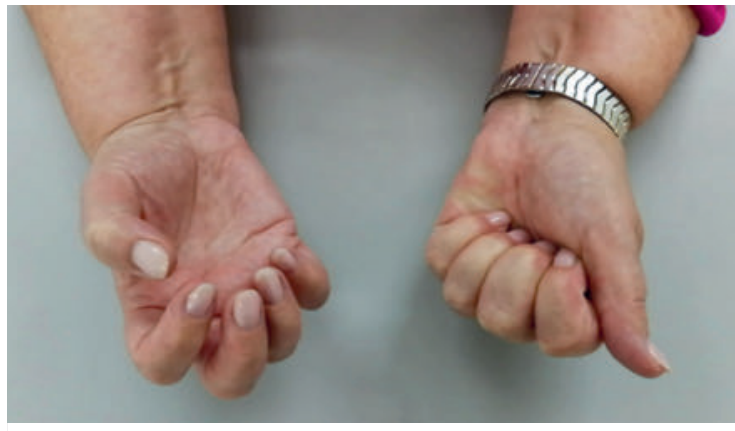

Abb. 2 Aktive Bewegungseinschränkung des Faustschlusses bei einem chronischen CRPS der rechten Hand. (Quelle: C. Storz, E. Kraft)

wird wohl noch einiges an Forschung notwendig sein, bis präzise Aussagen dazu getroffen werden.

Des Weiteren weisen neurologische Studien darauf hin, dass es bei CRPS zu Körperschemastörungen (veränderte Wahrnehmung der Form und/oder Größe der betroffenen Extremität) kommen kann. Ursache hierfür könnten Veränderungen verschiedener Teile der Großhirnrinde (somatosensorische, parietale, insulare und frontale Bereiche) sein [18][19][20][21]. Zusätzlich gibt es weitere Hinweise, dass diese Patienten die Position der betroffenen Extremität im Raum defizitär oder verzögert erkennen [22]. Auch dies kann sich negativ auf eine gesunde Körperwahrnehmung auswirken. Förderreuther und Kollegen fanden in einer Studie heraus, dass $48 \%$ der Patienten mit CRPS darin eingeschränkt waren, die Finger ihrer betroffenen Hand zu erkennen, wohingegen nur 6,5\% der Patienten Schwierigkeiten beim Erkennen ihrer Finger der nicht betroffenen Hand zeigten [23].

\section{Möglichkeiten der apparativen Diagnostik bei Veränderungen im Gehirn}

Der Einsatz von Standarddiagnostik zur Darstellung von möglichen Veränderungen im Gehirn ist nicht notwendig bei CRPS. Allenfalls für wissenschaftliche Zwecke können verschiedene strukturelle und funktionelle Verfahren eingesetzt werden. Eine Auflistung dieser Verfahren inklusive deren Erläuterungen sowie ihr bisheriger Einsatz in der CRPS-Forschung ist in Tabelle 1 aufgeführt ( Tab. 1).

\section{Up-Date Leitlinien}

Es existieren verschiedene Empfehlungen, die das Management und die Behandlung von Patienten mit CRPS erleichtern sollen. Folgende drei Empfehlungen sind aktuell:

- Britische Leitlinie „Complex regional pain syndrome in adults" [34]

- Deutsche S1-Leitlinie „Diagnostik und Therapie komplexer regionaler Schmerzsyndrome (CRPS)“ [9] 
\Tab.1 Apparative Diagnostik zur Erfassung von Gehirnveränderungen und deren Einsatz in der CRPS-Forschung.

\begin{tabular}{|c|c|c|}
\hline apparative Diagnostik & Beschreibung & Einsatz in der CRPS-Forschung \\
\hline \multicolumn{3}{|l|}{ strukturelle Verfahren } \\
\hline $\begin{array}{l}\text { Magnetresonanztomografie } \\
\text { (MRT)/Kernspintomografie }\end{array}$ & $\begin{array}{l}\text { computergestütztes bildgebendes Verfahren zur } \\
\text { strukturellen Abbildung von Gehirngewebe durch Ver- } \\
\text { änderungen eines Magnetfeldes }\end{array}$ & $\begin{array}{l}\text { selten: Erfassen von strukturellen Veränderungen im } \\
\text { Gehirn, z. B. Volumenveränderungen der grauen Substanz } \\
\text { [24] }\end{array}$ \\
\hline Computertomografie (CT) & $\begin{array}{l}\text { computergestütztes bildgebendes Verfahren zur } \\
\text { strukturellen Abbildung von Gehirngewebe, basierend auf } \\
\text { Röntgenstrahlung }\end{array}$ & $\begin{array}{l}\text { spielt bislang keine Rolle: im Rahmen von Studien bis } \\
\text { dato nur als Navigationshilfe für Therapieverfahren wie } \\
\text { Ganglien- oder Sympathikusblockaden und nicht für die } \\
\text { Darstellung von Gehirnveränderungen [25] }\end{array}$ \\
\hline \multicolumn{3}{|l|}{ funktionelle Verfahren } \\
\hline $\begin{array}{l}\text { funktionelle Magnetreso- } \\
\text { nanztomografie (fMRT) }\end{array}$ & $\begin{array}{l}\text { bildgebendes Verfahren zur Darstellung der aktivierten } \\
\text { Gehirnareale durch die Messung der Veränderung der } \\
\text { Gewebedurchblutung }\end{array}$ & $\begin{array}{l}\text { häufig: wurde bereits mehrmals eingesetzt, um Ver- } \\
\text { änderungen der Topografie oder Verarbeitungsprozesse } \\
\text { des Gehirns nachzuweisen [26][27] }\end{array}$ \\
\hline $\begin{array}{l}\text { Single Photon-Emissions- } \\
\text { Computer-Tomografie } \\
\text { (SPECT) }\end{array}$ & $\begin{array}{l}\text { nuklearmedizinisches Verfahren zum Nachweis von Stoff- } \\
\text { wechselprozessen im Gehirn, basierend auf Röntgenstrah- } \\
\text { lung inklusive Injektion radioaktiv markierter Substanzen }\end{array}$ & $\begin{array}{l}\text { selten: wurde bisher nur in einer Einzelfall-Studie bei } \\
\text { CRPS nach Apoplex verwendet, um den Stoffwechsel des } \\
\text { Thalamus darzustellen [28] }\end{array}$ \\
\hline $\begin{array}{l}\text { Positronen-Emissions- } \\
\text { Tomografie (PET) }\end{array}$ & $\begin{array}{l}\text { nuklearmedizinisches bildgebendes Verfahren zum Nach- } \\
\text { weis von Stoffwechselprozessen im Gehirn, basierend auf } \\
\text { Röntgenstrahlung inklusive Injektion radioaktiv markierter } \\
\text { Substanzen }\end{array}$ & $\begin{array}{l}\text { selten: Darstellung von Glukose-Stoffwechsel im Bereich } \\
\text { der somatosensorischen Wahrnehmung des Gehirns bei } \\
\text { CRPS [29] }\end{array}$ \\
\hline $\begin{array}{l}\text { Magnetenzephalografie } \\
\text { (MEG) }\end{array}$ & $\begin{array}{l}\text { bildgebendes nicht invasives Verfahren zur Messung der } \\
\text { zeitlichen Magnetfeldveränderungen an der Kopfoberfläche }\end{array}$ & $\begin{array}{l}\text { häufig: wurde mehrfach eingesetzt, um kortikale Prozes- } \\
\text { se nach einem Stimulus oder allgemein Veränderungen } \\
\text { im Gehirn sichtbar zu machen [30][31][32] }\end{array}$ \\
\hline \multicolumn{3}{|c|}{ Verfahren zur Erfassung elektrischer Hirnaktivität } \\
\hline $\begin{array}{l}\text { Elektroenzephalografie } \\
\text { (EEG) }\end{array}$ & $\begin{array}{l}\text { Verfahren zur Erfassung von Aktivitätszuständen des } \\
\text { Gehirns durch die Ableitung von elektrischen Potenzial- } \\
\text { schwankungen von Nervenzellverbänden im Gehirn mittels } \\
\text { mehrerer Kopfhaut-Elektroden }\end{array}$ & $\begin{array}{l}\text { häufig: wurde mehrfach eingesetzt, um evozierte } \\
\text { Potentiale (Potentialunterschiede nach Reizung eines } \\
\text { Nervs oder Sinnesorgans) nach somatosensibler Reizung } \\
\text { abzuleiten [33] }\end{array}$ \\
\hline
\end{tabular}

- Empfehlungen der European Pain Federation "Standards of the diagnosis and management of CRPS“ [35]

In allen drei Leitlinien ist die Behandlung der Betroffenen auf hauptsächlich drei Pfeilern aufgebaut: die pharmakologische, die psychotherapeutische und die rehabilitative Therapie. Ebenfalls betonen diese Leitlinien einen multidisziplinären Behandlungsansatz innerhalb des biopsychosozialen Modells. Die aktive Beteiligung des Patienten ist nach Ansicht der Autoren der maßgebliche Indikator für eine erfolgreiche Therapie. Interventionelle Therapien hingegen sollten laut der deutschen S1-Leitlinie nur bei therapierefraktären Patienten zur Anwendung kommen. Eine detailliertere Auflistung mit den jeweiligen Empfehlungen ist in Tabelle 2 aufgeführt ( $\triangleright$ Tab. 2). Wichtig ist jedoch, dass die interventionellen Therapien immer in erfahrenen spezialisierten Schmerzzentren durchgeführt werden sollen.

\section{Die Rolle der topischen medikamentösen Therapie}

Die Anwendung der topischen medikamentösen Therapie wie Dimethylsulfoxid (DMSO) oder Capsaicin wird kontrovers diskutiert.

\section{Dimethylsulfoxid (DMSO)}

DMSO wirkt, indem es die Haut penetriert und freie Radikale einfängt, die durch Ischämien oder Entzündungen verursacht werden. Es gibt 2 Studien, die zur Behandlung von CRPS eine 50 \%ige DMSO-Creme 5 -mal täglich verwendet haben [36][37]. Aufgrund der nicht komplett transparenten Studienabläufe haben sich die Autoren der deutschen S1-Leitlinine dazu entschlossen, dass die Ergebnisse nicht eindeutig interpretierbar sind [9]. Obwohl DMSO in den Niederlanden zur Standardtherapie für CRPS zählt, kann aufgrund der Datenlage keine klare Empfehlung ausgesprochen werden. Zusätzlich kann es zu Nebenwirkungen, z. B. lokalen Hautirritationen, kommen. Auch die Möglichkeit der Karzinogenität von DMSO kann bis dato nicht komplett ausgeschlossen werden [9].

\section{Capsaicin}

Capsaicin ist ein pflanzliches Produkt, das aus der Chilischote extrahiert wird. Capsaicin verringert lokal zum einen die Reizweiterleitung von Nozizeptoren (durch C-Faser-Denervierung) und reizt zum anderen den Auftragungsort und fördert dort die Durchblutung und ein Wärmegefühl. 2001 wurde eine experimentelle EinzelfallStudie zu der Wirkung von 2-mal täglich 0,75\% Capsaicin bei akutem CRPS I der unteren Extremität in Kombination mit einem Mobilisationsschema mit zunehmender Ge- 
\Tab.2 Interventionelle Therapien bei CRPS und deren Empfehlung in der S1-Leitlinie [9].

\begin{tabular}{|c|c|c|}
\hline interventionelle Therapie & Indikation & Empfehlung der S1-Leitlinie \\
\hline Sympathikusblockade & $\begin{array}{l}\text { - bei sympathisch unterhaltendem Ruheschmerz } \\
\text { - nach positiven Testinjektionen }\end{array}$ & $\begin{array}{l}\text { aufgrund fehlender Evidenz nicht } \\
\text { empfohlen }\end{array}$ \\
\hline $\begin{array}{l}\text { rückenmarknahe Elektrostimulation } \\
\text { (SCS) }\end{array}$ & $\begin{array}{l}\text { - ohne mechanische Allodynie } \\
\text { - ohne gravierende psychische Erkrankung } \\
\text { - nach positiver Probestimulation }\end{array}$ & empfohlen nur für die untere Extremität \\
\hline $\begin{array}{l}\text { elektrische Stimulation von Spinal- } \\
\text { ganglien (DRG-Stimulation) }\end{array}$ & $\begin{array}{l}\text { - für die untere Extremität } \\
\text { - ohne gravierende psychische Erkrankung } \\
\text { - nach positiver Probestimulation }\end{array}$ & keine offizielle Empfehlung \\
\hline $\begin{array}{l}\text { repetitive transkranielle Magnet- } \\
\text { stimulation (rTMS) des Motorkortex }\end{array}$ & $\begin{array}{l}\text { - bestenfalls in klinischen Studien } \\
\text { - nur in spezialisierten Zentren }\end{array}$ & in Einzelfällen empfohlen \\
\hline $\begin{array}{l}\text { intrathekale Applikation von Wirk- } \\
\text { stoffen (Daten nur zu Baclofen) }\end{array}$ & $\begin{array}{l}\text { - nur bei schmerzhaften und therapielimitierenden } \\
\text { dystonen Störungen } \\
\text { - in Zentren, die mit der Methode Erfahrung haben }\end{array}$ & keine offizielle Empfehlung \\
\hline Plasmapherese & & nicht empfohlen \\
\hline peridurale und Plexus-Analgesie & & $\begin{array}{l}\text { gelten als eher experimentelle Verfahren; } \\
\text { keine Aussage darüber in der Leitlinie }\end{array}$ \\
\hline
\end{tabular}

wichtsbelastung durchgeführt [38]. Laut den Autoren verringerte sich die gemessene Schmerzstärke der Allodynie innerhalb von 3 Wochen. Jedoch kann auch bei dieser topischen Therapie aufgrund der geringen Studienlage und des Studiendesigns keine Empfehlung ausgesprochen werden. Darüber hinaus gibt es seit einigen Jahren ein Capsaicinpflaster mit $8 \%$, das in Einzelfällen bisher bei Patienten mit CRPS mit unterschiedlichem Erfolg eingesetzt worden ist [39][40]. Wichtig zu wissen ist, dass Capsaicin nach der Anwendung in der Anfangsphase der Behandlung zusätzlich brennende Schmerzen (vor allem bei der Pflasterapplikation) oder juckende Hautirritationen auslösen kann.

Eine detailliertere Ausführung und Kombinationsmöglichkeiten verschiedener Medikamente bei CRPS würden den Umfang dieses Artikels sprengen. Aus diesem Grund wurde hier nur auf die topische medikamentöse Therapie eingegangen.

\section{Fazit - Ausblick und neue Therapieformen}

Es ist weitere Forschung notwendig, um die Entwicklung und den Verlauf des CRPS weiter zu verstehen. Nichtsdestotrotz wird neben der Ätiologie, Pathogenese und medikamentösen Therapie auch an rehabilitativen Behandlungsmöglichkeiten geforscht. So stellen neue Therapievarianten, wie beispielsweise ein Handschuh zur sensiblen Stimulation der Fingerspitzen, vielversprechende Ansätze dar. Diese Stimulation soll plastische Veränderungen im Gehirn von Patienten mit CRPS bewirken [41]. Auch innovative Therapiemöglichkeiten, wie z. B. Exergames (computergestützte Spielsoftware für Bewegungen), werden von Patienten mit CRPS gut angenommen und bieten eine Möglichkeit, bereits frühzeitig zu aktiven Bewegungsübungen zu motivieren [42].
Autorinnen/Autoren

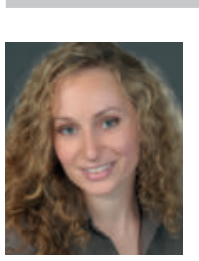

\section{Claudia Storz (M.Sc.)}

ist Ergotherapeutin und wissenschaftliche Mitarbeiterin der Forschungsgruppe Neurorehabilitation und Schmerz am LMU Klinikum München. Seit 2015 beschäftigt sie sich schwerpunktmäßig mit der Diagnostik und Behandlung des CRPS im klinischen Alltag sowie im Forschungsbereich.

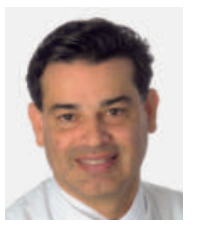

\section{Priv.-Doz. Dr. med. Eduard Kraft}

ist geschäftsführender Oberarzt der Klinik und Poliklinik für Orthopädie, Physikalische Medizin und Rehabilitation, Leiter der Interdisziplinären Schmerzambulanz Campus Großhadern und Leiter der Forschungsgruppe Neurorehabilitation und Schmerz am LMU Klinikum München.

Korrespondenzadresse

Claudia.Storz@med.uni-muenchen.de Eduard.Kraft@med.uni-muenchen.de

\section{Literatur}

Literaturverzeichnis am Ende der HTML-Version unter www.thieme-connect.de/products/manuelletherapie

\section{Bibliografie}

DOI https://doi.org/10.1055/a-1266-4361

manuelletherapie 2020; 24: 223-226

(c) 2020. Thieme. All rights reserved.

Georg Thieme Verlag KG, Rüdigerstraße 14, 70469 Stuttgart, Germany

ISSN 1433-2671 